\title{
La evaluación del riesgo del cliente dentro de la administración del capital de trabajo
}

\author{
Ana del Carmen Fernández Andrés ${ }^{1}$ \\ Profesora e investigadora de la Universidad de Camagüey \\ Cuba \\ João Nelito Mateus ${ }^{2}$ \\ Profesor y funcionario del Ministerio de Finanzas \\ Universidad Jean Piaget de Angola \\ Angola
}

Anisabel Gálvez Fernández ${ }^{3}$

Profesora e investigadora de la Universidad de Camagüey

Cuba

\section{Resumen}

En la actualidad el capital de trabajo representa el fundamental capital con que trabajan muchas empresas en el mundo, por lo cual es vital una eficiente administración del mismo. Dentro del capital de trabajo de la empresa una partida importante lo constituyen las cuentas por cobrar que se originan al

1 Licenciada en Economía. Máster en Economía. Doctora en Ciencias Económicas, especialidad Contabilidad y Finanzas. Profesora Titular de la Universidad de Camagüey, Cuba. E-mail: ana.fernandez@reduc.edu.cu

2 Licenciado en Economía, Máster en Economía, en la actualidad se encuentra realizando su doctorado en la Universidad la Matanza en Argentina, Profesor Asistente de la Universidad de Jean Piaget de Angola y funcionario del Ministerio de Finanzas. E-mail: jnelito2002@yahoo.es

3 Licenciada en Contabilidad y Finanzas, Máster en Contabilidad Gerencial, en la actualidad se encuentra realizando su doctorado en la Universidad de Camagüey, Cuba, Profesor Asistente de la Universidad de Camagüey. E-mail: anisabel.galvez@reduc.edu.cu

Recibido: 17/06/2015 - Versión final aceptada: 26/10/2015 
otorgar crédito comercial. El principal objetivo del administrador de crédito comercial consiste en ayudar a construir una amplia y creciente base de ventas rentables. Por tanto; el administrador de crédito comercial debe analizar las tendencias de ventas, del desempeño de la administración, de la posición de la liquidez, del apalancamiento y de la rentabilidad de los clientes actuales y potenciales de la empresa. El presente artículo sobre la administración financiera de las cuentas por cobrar dentro del capital de trabajo tiene como objetivo definir un procedimiento para la evaluación del riesgo del cliente que conlleve al establecimiento de estrategias crediticias.

Palabras clave: riesgo, cuentas por cobrar, capital de trabajo

\begin{abstract}
Currently, work capital represents the fundamental capital to work in many companies in the world, which is vital for an efficient administration. Inside the work capital of the company, the accounts receivable constitute an important item, originating in granting trade credit. The main objective of trade credit manager is to help build a broad and growing base of profitable sales. Therefore; commercial credit manager must analyze sales trends, the performance of the administration, the position of liquidity, leverage and profitability of existing and potential customers of the company. This article on the financial management of receivables inside the work capital aims to define a procedure for assessing customer risk that may lead to the establishment of credit strategies.
\end{abstract}

Key words: risk, accounts receivable, work capital

\title{
Introducción
}

Para que una empresa sea exitosa y pueda hacer frente a sus operaciones y emergencias sin peligro de una insolvencia técnica debe administrar eficientemente su capital de trabajo, ya que éste:

- Asegura en alto grado el mantenimiento del crédito de la empresa y provee lo necesario para hacer frente a emergencias

- Permite tener los inventarios a un nivel que capacita al negocio para producir en correspondencia a las necesidades de los clientes.

- Capacita a la empresa a otorgar condiciones de créditos favorables a sus clientes. 
- Habilita a la empresa a operar su negocio mediante el cumplimiento exitoso de su ciclo operativo y su ciclo de caja.

Si se parte que la mayoría de las empresas en el mundo son micro y pequeñas la situación planteada se agudiza, ya que estas empresas se caracterizan en general por:

- Ser negocios familiares.

- El ambiente empresarial, recurrentemente, se limita a controles enfocados a la salvaguarda de activos.

- Falta de funcionabilidad y operatividad de los controles establecidos.

- Limitada segregación de funciones.

- Cuenta con poca cantidad de empleados, muchas veces sin la calificación profesional requerida.

- No realizan procesos de identificación y evaluación de riesgos.

- Limitado acceso a las fuentes de financiamiento externa

- Limitada utilización de la tecnología de la información como parte de la gestión empresarial

- Administración espontánea

Lo que implica, en el contexto empresarial, sobre todo de este tipo de empresas, se manifiestan serias insuficiencias en la administración del capital de trabajo, tales como:

- Proceso de descapitalización del mismo

- No se realizan análisis sobre capital de trabajo que brinden los elementos necesarios y suficientes a la gerencia para la toma de decisiones

- No se realizan evaluaciones del riesgo del capital de trabajo

- Pobre gestión de inventarios

- Pobre gestión de cobros y pagos.

- No se trabaja con el capital de trabajo necesario en la empresa

Lo anterior, incide que no exista un control operativo en la administración del capital de trabajo, donde una de sus partidas más importante lo constituyen las cuentas por cobrar. 
Las cuentas por cobrar constituyen una de las partidas más importante del capital de trabajo, representan los importes de los productos, mercancías o servicios facturados y entregados a clientes que se encuentran pendientes de cobro, o sea constituyen derechos a corto plazo por ventas y servicios prestados, son créditos a cargo de clientes, que pueden convertirse en bienes o valores más líquidos disponibles y que por lo tanto pueden ser cobrados. En esta cuenta se reflejan todas las transacciones comerciales de la entidad por cualquiera de los servicios que brinda o productos que comercializan.

Es necesario que las empresas organicen y definan una política de crédito comercial que se convierta en la norma básica para la determinación, con un nivel de riesgo aceptable, si se debe conceder crédito a un cliente y las condiciones en que se va a conceder este, o sea plazos, instrumentos de pago, garantías, bonificaciones, penalizaciones, entre otras

Los niveles de las ventas y los cobros operan simultáneamente, ocasionando cambios en los costos del manejo de las cuentas por cobrar, lo cual ocurre también cuando se hacen cambios en las condiciones de créditos o en los procedimientos de cobros.

Por lo tanto, en correspondencia con la situación específica de cada cliente, debe establecerse y diferenciarse la política de crédito comercial a seguir con cada uno de ellos, por ende cuando se pretende vender a determinados clientes, para cobrar en el futuro, debe existir un margen de certeza de que este cliente es merecedor de esa confianza, o sea posee la dignidad de crédito necesaria para otorgarle un crédito comercial.

Lo planteado, da lugar a una contradicción entre la necesidad de una eficiente administración de las cuentas por cobrar, como partida importante del capital de trabajo de la empresa, y la ausencia de procedimientos contextualizados a nuestras realidades específicas, por lo cual el objetivo del presente artículo es, a partir del análisis del capital de trabajo y las cuentas por cobrar como importante componente de este, definir un procedimiento para la evaluación del riesgo del cliente que conlleve al establecimiento de estrategias crediticias. 


\section{Desarrollo}

Se realizó un estudio teórico sobre el capital de trabajo, donde se consultaron prestigiosos estudiosos del tema como Gitmán (2007) Westón (1995), Perdomo Moreno (2000), Marcelo Cruz Bustamante (2007), Mikel Ugando Peñate (2011), entre otros cuyos resultados se muestran a continuación:

Para el profesor Marcelo Cruz Bustamante (2007), el Capital Neto de Trabajo se define como la diferencia entre los activos circulantes y los pasivos a corto plazo, con que cuenta la empresa. Si los activos exceden a los pasivos se dice que la empresa tiene un capital neto de trabajo positivo. Por lo general cuanto mayor sea el margen por el que los activos circulantes puedan cubrir las obligaciones a corto plazo (pasivos a corto plazo) de la compañía, tanto mayor será la capacidad de esta para pagar sus deudas a medida que vencen.

Tal relación resulta del hecho de que el activo circulante es una fuente $\mathrm{u}$ origen de entradas de efectivo, en tanto que el pasivo a corto plazo es una fuente de salidas de efectivo.

Los desembolsos de efectivo que implican los pasivos a corto plazo son relativamente predecibles. Cuando la empresa contrae una deuda, a menudo se sabe cuándo vencerá esta.

Sin embargo para el profesor Lawrence Gitmán (2007) el Capital Neto de Trabajo también puede concebirse como la proporción de activos circulantes financiados mediante fondos a largo plazo, entendiéndose como fondos a largo plazo la suma de los pasivos a largo plazo y el capital social de una empresa.

Los profesores de la universidad mexicana de ANÁHAUC (2012), plantean:

El Capital de Trabajo

- Es el Activo Circulante, compuesto por:

- Caja y Banco,

- Cuentas por cobrar (clientes)

- Inventario (almacén). 
- Representa la porción de la inversión que circula de una forma a otra en la conducción ordinaria de la empresa (ciclo operativo de la empresa).

- Los pasivos circulantes representan el financiamiento a corto plazo, adeudos:

- a proveedores,

- a los bancos,

- a los empleados

- al gobierno.

Capital de trabajo neto

Se define como:

CTN $=$ Activos Circulantes - Pasivos Circulantes

Cuando AC>PC, el CTN $>0$

En este caso el CTN es la porción de activos circulantes de la empresa financiada con fondos a largo plazo.

\section{Cuando AC $<$ PC, el CTN $<0$}

En este caso (menos común) el CTN es la porción de los activos fijos de la empresa financiada con pasivos circulantes.

En general, cuanto mayor sea el margen con el que los activos circulantes cubren a sus pasivos circulantes, mayor será la capacidad para pagar sus cuentas conforme se vencen.

Peñate (2011) en su trabajo Evaluación financiera a corto plazo mediante la administración del capital de trabajo, nos brinda un grupo de definiciones y conceptos de capital de trabajo entre los cuales se encuentra:

- El capital de trabajo bruto se refiere simplemente a los activos circulantes.

- El capital de trabajo neto se define como los activos circulantes menos los pasivos circulantes. 
- El capital de trabajo es la inversión de una empresa en activos a corto plazo (efectivo, valores negociables, cuentas por cobrar e inventario).

Según la Red Interactiva de Estudiantes (RIIE 2010), procedente de la provincia San Luís, Argentina, se plantea que el término capital de trabajo es la inversión de una empresa en activos a corto plazo (efectivo, valores negociables, cuentas por cobrar e inventarios). Sergio Núñez (2011) del Instituto Mexicano Universitario de Tecnología Industrial y Administración Industrial nos plantea que el estudio del capital de trabajo es de mayor importancia para el análisis interno y externo, debido a su estrecha relación con las operaciones normales diarias de un negocio. Para este autor hay dos definiciones del capital de trabajo que parecen haber tenido una aceptación general:

- El capital de trabajo es el excedente del activo circulante sobre pasivo circulante. En otras palabras, el capital de trabajo representa el importe del activo circulante que no ha sido suministrado por los acreedores a corto plazo. Esta definición es de carácter cualitativo, puesto que muestra la posible disponibilidad del activo circulante en exceso del pasivo circulante; representa un índice de estabilidad financiera o margen de protección para los acreedores actuales y para futuras operaciones normales. La disponibilidad inmediata del capital de trabajo depende del tipo y de la naturaleza líquida de activos circulantes tales como caja, inversiones temporales en efectivo, cuentas por cobrar e inventarios. Cuando el capital de trabajo se define de esta forma, no puede aumentarse por medio de préstamos de los bancos o por medio de la ampliación de crédito por los acreedores.

- En el capital de trabajo es lo más importante el activo circulante. Esta interpretación es cuantitativa, puesto que representa el importe total de los recursos usados en las operaciones normales.

En esta definición el activo circulante se considera que es capital bruto de trabajo, y el excedente del activo circulante sobre el pasivo circulante es el capital neto de trabajo. El capital neto de trabajo representa el importe del activo circulante que quedaría si todo el pasivo circulante 
fuese pagado, suponiendo que no hubiese pérdida o ganancia al convertir el activo circulante en efectivo.

En el artículo El Capital de Trabajo, Giovanny E. Gómez (2005) define que el capital de trabajo puede entenderse como los recursos que una empresa utiliza para llevar a buen fin sus actividades operacionales.

Los recursos con que cuenta la gerencia son el disponible, las cuentas por cobrar y los inventarios, es decir, en términos contables, corresponden al activo corriente. Bajo la misma óptica contable, estos recursos se disminuyen por todo tipo de obligaciones de corto plazo que los comprometen, es decir, por los pasivos corrientes.

La diferencia entre activos corrientes y pasivos corrientes es los que, en términos de contabilidad, se denomina capital de trabajo.

$\mathrm{KT}=\mathrm{AC}-\mathrm{PC}$

Donde, $\mathrm{KT}$ = capital de trabajo

$\mathrm{AC}=$ activo corriente

$\mathrm{PC}=$ pasivo corriente

Erizbel Amat Álvarez (2012) brinda dos definiciones de capital de trabajo:

Capital de trabajo bruto: El cual constituye el total del activo circulante.

Capital de trabajo neto: Representa la diferencia entre el activo circulante y el pasivo circulante, o sea, refleja el importe del activo circulante que no ha sido suministrado por los acreedores a corto plazo. También es denominado Capital Circulante, Fondo Neto de Rotación, Fondo de Maniobra.

De lo anteriormente expuesto se infiere que el capital neto de trabajo o capital circulante es la parte de las fuentes permanentes que financian el Activo Circulante.

El estudio realizado nos ha permitido sistematizar la existencia de tres perspectivas en el tratamiento de del capital de trabajo en la bibliografía especializada hasta la actualidad:

La contable o diferencia entre AC y PC que puede denominarse capital de trabajo neto contable. La gerencial en la que el capital de trabajo 
(KT) lo constituyen los recursos que una empresa requiere para cumplimentar su ciclo operativo. La financiera que representa aquella parte del activo circulante financiada con fondos a largo plazo.

A nuestro criterio cualquier acercamiento al capital de trabajo debe evaluar las tres perspectivas por lo cual se elabora la siguiente definición que contempla este enfoque integrador para definir el capital de trabajo: El capital de trabajo representa aquella parte del activo corriente que es financiada con fondos a largo plazo cuyo objetivo está dirigido a cumplimentar el ciclo operativo de la empresa, expresado en la ecuación AC menos PC.

En relación a la administración del capital de trabajo existe consenso autoral en asumirla como el manejo de las cuentas corrientes de la empresa que incluyen activos y pasivos circulantes, garantizando que los activos circulantes de la empresa sean suficientes para cubrir sus pasivos circulantes y poder así asegurar un margen de seguridad razonable, siendo su objetivo manejar cada uno de los activos y pasivos a corto plazo de manera que se alcance un nivel adecuado de capital de trabajo neto.

Como se ha planteado, las cuentas por cobrar constituyen parte del capital de trabajo de la empresa y las mismas representan el crédito comercial que concede la empresa a sus clientes con una cuenta abierta. Son derechos exigibles provenientes de ventas, servicios prestados, préstamos o anticipos otorgados; por tanto también hay que analizarlos y mostrarlos por los conceptos que lo motivaron; clientes, empleados, otras entidades, etc. Para conservar los clientes actuales y atraer nueva clientela, la mayoría de las empresas comprenden que es necesario ofrecer crédito comercial, lo que fundamenta que en la mayoría de las empresas las cuentas por cobrar representan gran parte de sus activos circulantes, por lo que normalmente se presta mucha atención a la administración eficiente de estas cuentas; ya que representan una inversión cuantiosa por parte de la mayoría de las empresas, por tanto su administración eficiente puede representar ahorros considerables. El objetivo general con respecto a la administración de las cuentas por cobrar debe ser, no sólo, el de cobrarla con prontitud; también debe 
prestarse atención a las alternativas de costo - beneficio que presentan en los diferentes campos de estas cuentas; pero específicamente es el de aumentar las ventas rentables al extenderle crédito comercial a los clientes dignos.

El administrador de crédito comercial debe desarrollar un archivo en el que reúna la información de crédito de cada cuenta; con el objetivo de evaluar y reevaluar la dignidad de crédito comercial de los clientes actuales y potenciales. Al evaluar requisitos de crédito comercial a corto plazo, el énfasis caerá sobre la liquidez sin embargo, un buen analista de crédito comercial también observará los factores a largo plazo, como son la estabilidad financiera, la capacidad de endeudamiento y la rentabilidad; ya que si el panorama a largo plazo parece tener un buen potencial, puede valer la pena que el acreedor se arriesgue con un cliente potencial cuya posición actual de liquidez sea débil, lo que a nuestro criterio, implica, asumir la administración de las cuentas por cobrar con un enfoque de riesgo.

Pero, ¿qué se asume como el riesgo del crédito comercial o de las cuentas por cobrar? A nuestro criterio, el riesgo del crédito comercial o de las cuentas por cobrar es la probabilidad que el cliente no pague por la mercancía vendida y no cobrada o pague fuera de los términos pactados provocando morosidad en la cobranza de la empresa con la correspondiente afectación financiera a la liquidez de la misma.

Por lo cual, las empresas deben diseñar procedimientos adecuados a sus realidades concretas que les permitan evaluar el riesgo del cliente, determinando si se le concede crédito comercial o no a este.

En la literatura especializada se referencian diferentes métodos, entre los cuales están:
a. Métodos de las $5 \mathrm{C}$.
b. Métodos de las $8 \mathrm{C}$.
c. Método de la capacidad financiera mínima.
d. Método de puntuaciones
e. Método de índice de riesgo 
a. Método de las 5 C: Es el procedimiento de la gerencia de crédito para evaluar y determinar, si se le concede crédito a un cliente, a través de los siguientes factores:

- Carácter: Factor de índole moral que valora la moralidad del cliente para cumplir con sus obligaciones.

- Capacidad: Habilidad del cliente para cumplir con sus pagos.

- Capital: Análisis de la situación financiera del cliente aplicando razones.

- Colaterales: Activos que pueda tener el cliente que sirvan de garantía del crédito.

- Condiciones: El entorno legal, político y económico en que se desenvuelve el cliente.

b. Método de la 8 C: $\mathrm{Al}$ método de las $5 \mathrm{C}$ se le agregan tres factores más:

- Cobertura: Seguros que pueda tener el cliente.

- Consistencia: Años de permanencia en el giro comercial.

- Categoría: Clasificación del cliente en: (bueno, regular, malo, nuevo, dudoso).

c. Método de la capacidad financiera mínima: Es un procedimiento de la gerencia de crédito para evaluar al cliente el cual el empresario establece los niveles mínimos que deben cumplir los mismos para otorgarles crédito comercial en cuanto a:

- Razón circulante que tiene como significado medir la capacidad del negocio para pagar sus obligaciones en el corto plazo; es decir, la capacidad que tiene la empresa de pagar las deudas de su pasivo circulante con los recursos que tiene en activos circulantes. Se calcula tomando el total de los activos circulantes del balance general y se divide entre el total de pasivos circulantes:

$$
\text { Razón circulante }=\frac{\text { Activos circulante }}{\text { Pasivo circulante }}
$$

- Margen de seguridad: No es más que la relación entre el fondo de maniobra y el pasivo circulante donde: 


$$
\text { Margen de seguridad }=\frac{\text { Fondo de maniobra }}{\text { Pasivo circulante }}
$$

d. Método de puntuaciones: Se aplica cuando la empresa tiene una clientela pequeña que puede ser investigada por el propio analista de cobranza. Este método consiste en la elaboración de un cuestionario que es respondido por el propio cliente en su visita a la empresa, donde se reflejan factores críticos que puedan poner en riesgo la cobranza. El gerente de crédito comercial establece una ponderación de puntos por cada factor de acuerdo a su nivel de importancia, la suma de los puntos de todos los factores debe dar 100 y se establece un nivel de puntaje para acreditar dignidad de crédito y a partir de este se otorga crédito.

e. Método de índice de riesgo: A partir del método de puntuaciones se establece un índice de calidad crediticia mediante la aplicación de la técnica estadística del análisis discriminante múltiple (ADM).

$\mathrm{Al}$ analizar los métodos más utilizados para la evaluación del riesgo del cliente se considera que el método del índice de riesgo a partir de que su base es el método de puntuaciones que permite tener presente las particularidades empresariales, a la posibilidad de su procesamiento informático que reduce el tiempo de cálculo y por ende el administrador financiero de las cuentas por cobrar pueda dedicar más tiempo al análisis sería una buena opción para las gerencias empresariales.

A partir de lo cual, los autores del presente trabajo definen al procedimiento para la evaluación del riesgo del cliente como el conjunto de operaciones que a partir del método de puntuaciones establece un índice de calidad crediticia mediante la aplicación de la técnica estadística del análisis discriminante múltiple $(\mathrm{ADM})$ que le permite a la empresa la diferenciación de su estrategia crediticia.

En correspondencia a lo anterior se plantea una estructura del procedimiento metodológico conformada por etapas, pasos y técnicas que se muestran a continuación: 


\section{ETAPA I. IDENTIFICACIÓN DE LAS VARIABLES A EVALUAR A LOS CLIENTES}

\section{PASOS:}

- Creación del grupo de expertos

- Selección de las variables por expertos

- Determinación de la concordancia de los expertos

- Jerarquización de las variables

\section{TÉCNICAS:}

- Creación del grupo de expertos

Los expertos se seleccionaran mediante el coeficiente de competencia a través de la fórmula:

$\mathrm{K}=0,5(\mathrm{Kc}+\mathrm{Ka})$

Dónde:

$\mathrm{K}=$ Coeficiente de Competencia.

Kc. $=$ Coeficiente de Conocimiento.

$\mathrm{Ka}=$ Coeficiente de Argumentación.

a) Coeficiente de Conocimiento $(\mathrm{Kc})$.

Se plantea la autoevaluación de los expertos en una escala de 1 a 10 de tendencia progresiva del nivel de conocimiento sobre el tema con el siguiente cuestionario:

Tabla 1. Evaluación del nivel de conocimiento.

\begin{tabular}{|l|l|l|l|l|l|l|l|l|l|l|}
\hline $\mathrm{N}^{\circ}$ Experto & 1 & 2 & 3 & 4 & 5 & 6 & 7 & 8 & 9 & 10 \\
\hline & & & & & & & & & & \\
\hline
\end{tabular}

Fuente: Elaboración propia a partir de la bibliografía consultada 
Se determina el coeficiente de conocimiento a partir de la fórmula que plantea:

$\mathrm{Kc}=($ nivel marcado por el experto X 0,1$)$

b) Coeficiente de Argumentación (Ka).

Se parte de la autoevaluación realizada por cada experto a través del cuestionario elaborado a partir de Campestrous L, Rizo. C, Indicadores e investigaciones educativas, La Habana, 2002, (formato electrónico) que establecen evaluar fundamentalmente: el nivel de preparación técnico profesional en el tema, el conocimiento del objeto de estudio y la experiencia profesional, el cual se muestra a continuación:

Tabla 2. Evaluación del nivel de argumentación.

\begin{tabular}{|l|l|l|l|}
\hline Fuentes de Argumentación & Alto & Medio & Bajo \\
\hline $\begin{array}{l}\text { Conocimientos del sector como resultado de } \\
\text { los años de experiencia del mismo }\end{array}$ & & & \\
\hline $\begin{array}{l}\text { Años de experiencia en el ejercicio de la } \\
\text { profesión de su puesto de trabajo }\end{array}$ & & & \\
\hline $\begin{array}{l}\text { Conocimientos técnicos de la categoría cuentas } \\
\text { por cobrar y evaluación del riesgo del cliente }\end{array}$ & & & \\
\hline $\begin{array}{l}\text { Estudios realizados sobre el tema de riesgos y } \\
\text { riesgos de control interno }\end{array}$ & & & \\
\hline $\begin{array}{l}\text { Posibilidades de identificación de las variables } \\
\text { que inciden en el nivel de riesgos de los clientes } \\
\text { de la empresa }\end{array}$ & & & \\
\hline
\end{tabular}

Fuente: Elaboración propia a partir de la bibliografía consultada

Para evaluar el nivel de argumentación de los expertos se elaboró la Tabla Patrón de Comparación que se muestra a continuación considerando los siguientes criterios:

Para realizar las ponderaciones de los puntos a las respuestas dadas por los expertos se asumirán los siguientes supuestos: 
1. La sumatoria de las respuestas a las preguntas que califiquen el nivel más alto no debe ser superior a 1 .

2. La importancia para la distribución de las puntuaciones por preguntas debe potenciar el cumplimiento del siguiente orden de jerarquía: Nivel de preparación técnico profesional en el tema, conocimiento del objeto de estudio como resultado de la experiencia profesional y práctica y el resto de los factores menos puntuación.

Como resultado se obtuvo la tabla que se muestra a continuación:

Tabla 3. Tabla patrón de comparación

\begin{tabular}{|l|c|c|c|}
\hline \multicolumn{1}{|c|}{ Fuentes de Argumentación } & Alto & Medio & Bajo \\
\hline $\begin{array}{l}\text { Conocimientos del sector como resultado de } \\
\text { los años de experiencia del mismo }\end{array}$ & 0.3 & 0.2 & 0.1 \\
\hline $\begin{array}{l}\text { Años de experiencia en el ejercicio de la } \\
\text { profesión de su puesto de trabajo }\end{array}$ & 0.05 & 0.05 & 0.05 \\
\hline $\begin{array}{l}\text { Conocimientos técnicos de la categoría cuentas } \\
\text { por cobrar y evaluación del riesgo del cliente }\end{array}$ & 0.5 & 0.4 & 0.3 \\
\hline $\begin{array}{l}\text { Estudios realizados sobre el tema de riesgos y } \\
\text { riesgos de control interno }\end{array}$ & 0.10 & 0.10 & 0.10 \\
\hline $\begin{array}{l}\text { Posibilidades de identificación de las variables } \\
\text { que inciden en el nivel de riesgos de los clientes } \\
\text { de la empresa }\end{array}$ & 0.05 & 0.05 & 0.05 \\
\hline
\end{tabular}

Fuente: Elaboración propia a partir de la bibliografía consultada

Los niveles de Competencia asumidos fueron:

Alta: $0,8 \leq \mathrm{K} \leq 1,0$

Medio: $0,5 \leq \mathrm{K}<0,8$

Bajo: $\mathrm{K}<0,5$

Con los resultados obtenidos se seleccionan los expertos a participar en la identificación. 
- Selección de las variables por expertos

Se le solicitará a cada experto que escriba las variables que a su criterio inciden en el nivel de riesgos de los clientes de la empresa, asumiéndose el nivel de riesgo del cliente como la posibilidad que este no pague o pague fuera del término pactado

- Determinación de la concordancia entre los expertos

Se le circula los resultados de cada experto al resto de los expertos pidiéndole si están de acuerdo o no, marcando con una X su concordancia.

A estos resultados se les calcula el nivel de concordancia mediante la determinación del coeficiente de Kendal, este coeficiente mide el grado de concordancia entre un grupo de elementos $(\mathrm{K})$ y un grupo de características (n).La respuesta es ordinal. La hipótesis nula es que no hay concordancia: $\mathrm{W}=0$; $y$ la Hipótesis Alternativa es que si la hay $(\mathrm{W}>0)$. Este estadístico sigue una $\chi 2$ con grados de libertad: $n-1$, mediante la siguiente fórmula:

$$
r=\frac{N_{1}+N_{-1}}{\sqrt{\left[\frac{1}{2} n(n-1)-t^{\prime}\right]\left[\frac{1}{2}\left(n(n-1)-u^{\prime}\right]\right.}} \quad t^{\prime}=\frac{1}{2} \sum_{t} t(t-1) \quad u^{\prime}=\frac{1}{2} \sum_{u} u(u-1)
$$

Dónde:

$\mathrm{n}$ es la cantidad de expertos

$\mathrm{N}_{1}$ es el total con semejanza

$\mathrm{N}_{-1}$ total con desemejanza

t es el número de ligas en la categoría (empates)

u es el número de ligas en otras categorías (empates)

Si el coeficiente no fuera significativo será necesario proceder a otra ronda de consulta hasta que se logre el consenso deseado.

Se determina el criterio predominante de acuerdo a los expertos. 
- Lerarquización de las variables

Para la jerarquización, según su nivel de importancia, de las variables ya identificadas y consensadas se diseñan los siguientes instrumentos para aplicar a los expertos.

Se le pedirá a cada experto que marque en una escala ascendente de 10 a 100 puntos el nivel de importancia que le concede a cada variable identificada en la siguiente tabla:

Tabla $\mathrm{N}^{\circ}$ 5: Jerarquización de las variables por experto

\begin{tabular}{|l|l|l|l|l|l|l|l|l|l|l|}
\hline $\begin{array}{c}\text { Variables } \\
\text { identificadas }\end{array}$ & 100 & 90 & 80 & 70 & 60 & 50 & 40 & 30 & 20 & 10 \\
\hline 1 & & & & & & & & & & \\
\hline. & & & & & & & & & & \\
\hline$M$ & & & & & & & & & & \\
\hline
\end{tabular}

Fuente: Elaboración propia a partir de la bibliografía consultada

Posteriormente se determina la moda, elaborándose la siguiente tabla

Tabla 6. Jerarquización de las variables

\begin{tabular}{|l|l|l|}
\hline Variables identificadas & \multicolumn{1}{|c|}{ Moda } & \multicolumn{1}{c|}{ Prioridad } \\
\hline 1 & & 1 \\
\hline$\cdot$ & & $\cdot$ \\
\hline $\mathrm{n}$ & & $\mathrm{m}$ \\
\hline
\end{tabular}

Fuente: Elaboración propia a partir de la bibliografía consultada 


\section{ETAPA II. ELABORACIÓN DEL CUESTIONARIO}

\section{PASOS:}

- Elaboración del cuestionario a partir de las variables identificadas, concordadas y jerarquizadas

\section{TÉCNICAS}

Se aplicará la técnica de cuestionario donde se elaborará un conjunto de preguntas respecto a las 5 variables más importantes identificadas por los expertos que determinan en el nivel de riesgo de los clientes. Se realizaran preguntas cerradas y las posibilidades de respuesta se asumirán en una escala de Likert, en el intervalo de 1 a 4, donde 1 es considerada la peor situación; por lo tanto 4 es la más satisfactoria. Para asignar este puntaje se establece:

1: No

2: A veces

3: Casi siempre

4: $\mathrm{Si}$

\section{ETAPA III. DETERMINACIÓN DE LOS INDICES DE CALIDAD CREDITICIA}

\section{PASOS:}

- Ponderación de puntos por preguntas del cuestionario

- Determinación de los rangos de calidad crediticia

\section{TÉCNICAS}

Se realizará la ponderación de puntos de la siguiente manera: 


\begin{tabular}{|l|l|}
\hline \multicolumn{1}{|c|}{ POSIBILIDAD DE RESPUESTA } & \multicolumn{1}{c|}{ PUNTAJE } \\
\hline 1: No & 5 \\
\hline 2: A veces & 10 \\
\hline 3: Casi siempre & 15 \\
\hline 4: $\mathrm{Si}$ & 20 \\
\hline
\end{tabular}

Fuente: Elaboración propia a partir de la bibliografía consultada

Para la determinación de los rangos de calidad crediticia se realizara la sumatoria de los puntajes según las opciones de respuesta a las preguntas, estableciéndose los rangos siguientes:

$$
\begin{aligned}
& \sum_{i=1}^{n=2} P=\text { CLIENTES MALOS } \\
& \sum_{i=2}^{n=3} P=\text { CLIENTES REGULARES } \\
& \sum_{i=3}^{n=4} P=\text { CLIENTES BUENOS }
\end{aligned}
$$

Se aplicará la técnica de análisis discriminante múltiple para confirmar los rangos de calidad crediticia establecidos

\section{ETAPA IV. DEFINICIÓN DE LAS ESTRATEGIAS CREDITICIAS}

\section{PASOS:}

- Categorización de los clientes

- Determinación de la estrategia crediticia por tipo de cliente 


\section{TÉCNICAS}

Mediante trabajo en grupo con la gerencia de crédito comercial de la empresa se determinaran la categorización de los clientes y la estrategia crediticia por tipo de clientes siguientes:

1. Clientes buenos: clientes que regularmente cumplen sus pagos con la empresa en los términos establecidos y cuando no pueden pagar en los términos establecidos renegocian sus deudas.

2. Clientes regulares: clientes que regularmente cumplen sus pagos con la empresa en los términos establecidos y cuando no pueden pagar en los términos establecidos no renegocian sus deudas oportunamente.

3. Clientes malos: clientes que pocas veces cumplen sus pagos con la empresa en los términos establecidos.

El procedimiento ha sido aplicado en una empresa del giro alimentario, donde se elaboró la siguiente estrategia crediticia:

\section{Clientes buenos}

- Se le vende a crédito sin límite de ventas.

- Se le otorgan plazos flexibles de crédito de 60 hasta 90 días

- Pagan con cualquier instrumento de pago de los establecidos en la legislación vigente

- No se establecen sanciones ante demora o incumplimiento del pago.

2. Clientes regulares

- Se le vende a crédito hasta un límite de ventas mensuales de 10 a 50 mil pesos.

- Se le otorgan plazos de crédito de 30 hasta 60 días

- Pagan con los siguientes instrumento de pago de los establecidos en la legislación vigente: cheques y transferencias

- Se establecen sanciones ante demora o incumplimiento del pago siguientes: de $0.05 \%$ de recargo por mora por cada día de demora sobre lo facturado, se le limita el crédito a 30 días y se le disminuye el límite de ventas a crédito en un $10 \%$ 
3. Clientes malos:

- No se le otorgan ventas a créditos.

$\mathrm{Al}$ aplicarse esta estrategia crediticia se logra una disminución de la morosidad de las cuentas por cobrar significativo, la transición de un grupo representativo de clientes inicialmente categorizados como malos y regulares a categorías superiores de dignidad crediticia y una mejor estructura del envejecimiento por edades de las cuentas por cobrar.

Estos resultados han provocado una disminución del monto promedio inmovilizado en cuentas por cobrar y por ende del costo oportunidad de las mismas.

\section{Conclusiones}

1. El estudio realizado permite plantear que constituye una necesidad el diseño de procedimientos de evaluación del riesgo del cliente adecuado a nuestras particularidades.

2. El método del índice de riesgo a partir de que su base es el método de puntuaciones que permite tener presente las peculiaridades empresariales, a la posibilidad de su procesamiento informático que reduce el tiempo de cálculo y por ende el administrador financiero de las cuentas por cobrar pueda dedicar más tiempo al análisis se considera una buena opción para las gerencias empresariales.

\section{Bibliografía}

Ávila Díaz, G.l. (2001). Análisis de las Cuentas por Cobrar. Tesis de Maestría, Universidad de Camagüey, Camagüey, Cuba

Amat, E. . (2012). El análisis económico financiero como herramienta de dirección. Generalidades. Recuperado el 22 de mayo del 2014, de http://www. gestiopolis.com/.../analisis - economico - financiero-1.html

Anahauc, E. (2012, 24 de julio). Capital de trabajo. [en línea]. México: Universidad ANAHAUC. Recuperado el 20 de mayo del 2014, de http://www. anahuaoakaca.edu.mx 
Brigham, M. E. (2007). Finanzas corporativas. (2da ed.). México: Editorial Thomson.

Bustamante, M. C. (2007, 22 de mayo). Instituto de Estudios Bancarios Guillermo Subercaseaux. Recuperado el 12 de marzo del 2014, de www.ieb.ch

Durán, Y. (2011, Enero - Junio). Administración del Capital de Trabajo: una herramienta financiera. Visión Gerencial, 10 (1), 37-56.

Estudiantes, R. I. (18 de abril de 2010). Administración del capital de trabajo. Recuperado el 15 de febrero de 2014, de http://www.uba.ar

Fernández, A. (2012). La Administración Financiera del Capital de Trabajo. Tesis de Maestría, Inédito, Universidad de Camagüey, Camagüey, Camagüey, Cuba

Fred Weston, T. C. (1995). Finanzas en administración. (9na ed.). México: Editorial McGraw Hill.

Gitmán, L. (2007). Principios de Administración Financiera. (11na ed.). México: Prentice Hall.

Gómez, G. E. (2005). Administración del capital de trabajo. Recuperado el 15 de febrero de 2014, Conshttp://www.gestiopolis.com/canales/financiera/ articulos/no\%205/administracioncapitaltrabajo.htm

Najul, M. (2009, octubre - diciembre). Insolvencia necesaria del capital de trabajo. Revista Debates IESA, XIV( 4), 17-20.

Nuñez, S. (2011). Capital neto de trabajo. Recuperado el 3 de marzo de 2014, de http://www.buenastareas.com/ensayos/Capital-de-Trabajo/1482744.html

Pascual, J. (1992). Análisis de la empresa a través de su información económico-financiera. Madrid: Editorial Pirámide S.A.

Peñate, M. U. (2011, 25 de septiembre). Evaluación Financiera a corto plazo mediante la administración del capital de trabajo. Recuperado el 2 der marzo de 2014, de http://ww.gestiopolis.com/recursos/documentos/folletos/ fin/...

Perdomo Moreno, A. (2000). Conceptos básicos de administración financiera. México: Editorial PEMAS.

S. Ross, R. W. (2009). Finanzas corporativas. (9na ed.). México: Editorial McGraw- Hill. 